\title{
Relativistic Configuration Interaction Calculation of the Correlation Energies of Heliumlike Ions
}

\author{
Kwok-Tsang Cheng \\ Mau Hsiung Chen \\ W.R. Johnson
}

This paper was prepared for submittal to the Second International Conference on Computational Physics, Beijing, China September 12-18, 1993

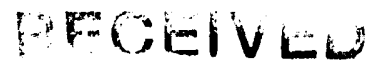

AUG 021933

September 1993

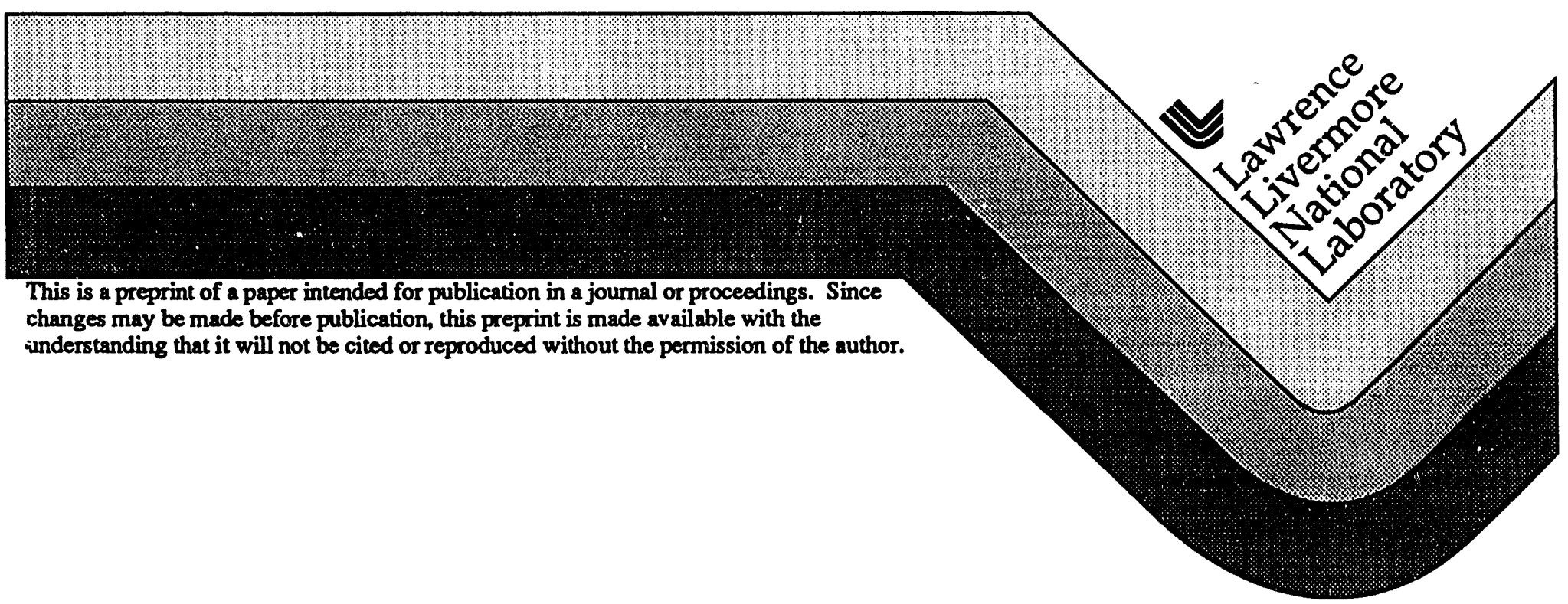




\section{DISCLAIMER}

This document was prepared as an account of work sponsored by an agency of the United States Government. Neither the United States Government nor the University of California nor any of their employees, makes any warranty, express or implied, or assumes any legal liability or responsibility for the accuracy, completeness, or usefulness of any information, apparatus, product, or process disclosed, or represents that its use would not infringe privately owned rights. Reference herein to any specific commercial products, process, or service by trade name, trademark, manufacturer, or otherwise, does not necessarily constitute or imply its endorsement, recommendation, or favoring by the United States Government or the University of California. The views and opinions of authors expressed herein do not necessarily state or reflect those of the United States Government or the University of California, and shall not be used for advertising or product endorsement purposes. 


\title{
Relativistic Configuration Interaction Calculation of the Correlation Energies of Heliumlike Ions ${ }^{1}$
}

\author{
Kwok-Tsang Cheng and Mau Hsiung Chen \\ University of California, Lawrence Livermore National Laboratory \\ Livermore, California 94550, USA \\ W. R. Johnson \\ Physics Department, University of Notre Dame, Notre Dame, Indiana 46556, USA
}

\begin{abstract}
In this paper, we present a new relativistic configuration interaction (CI) method for atomic structure calculations using finite basis sets constructed from B spline functions of piecewise polynomials. This method is used to calculate the correlation energies of the low-lying states of two-electron heliumlike ions. Our calculations are based on the relativistic no-pair Hamiltonian from quantum electrodynamics (QED). Eigenfunctions of the Hamiltonian are expanded in a basis of two-electron configuration state functions (CSF) constructed from products of one-electron orbitals. Eigenvalues and eigenfunctions of the low-lying two-electron states are then determined by diagonalizing the Hamiltonian matrix as in the usual $\mathrm{CI}$ approach.

The one-electron basis orbitals are B spline solutions of the Dirac equation for an electron in a Coulomb field constrained to a finite cavity [1]. Boundary condition is imposed by the MIT bag model. The B spline basis is finite and complete. The resulting modified Dirac spectrum consists of a finite set of discrete eigenstates which separate cleanly into positive- and negativeenergy spectra. The positive-energy branch contains terms that belong to the bound-state spectrum as well as the positive-energy continuum of the unmodified Dirac spectrum. As a result, the use of this B spline basis set in a CI calculation effectively includes interactions with the bound states as well as with the positive-energy continuum. Furthermore, by including only the positive-energy solutions of the modified Dirac equation in our CI calculations, positive-energy projection operators in the relativistic no-pair Hamiltonian are implicitly included.
\end{abstract}

\footnotetext{
${ }^{1}$ The work of KTC and MHC was performed under the auspices of the U.S. Department of Energy by Lawrence Livermore National Laboratory under contrect No. W-7405-ENG-48. The work of WRJ was supported by NSF grant PHY-92-04089.
} 
The CI equation leads to a large symmetric eigenvalue problem (dimension 5000 to 8000 ). The Hamiltonian matrix is dense, in contrast with the molecular or nuclear cases, where the matrix is often much larger but sparse. The eigenvalues and eigenvectors for the lowest few states are determined using the Davidson's method [2]. The evaluation of the Hamiltonian matrix is computationally intensive. Efficient algorithms have been developed to speed up the calculations of angular coefficients and Coulomb and Breit integrals by at least an order of magnitude. Even so, thirty to forty hours of CRAY-YMP supercomputer time are required for each heliumlike ion considered.

We have calculated the term energies for the $n=2$ triplet states of heliumlike ions with our relativistic CI approach. Mass polarization and QED corrections are obtained from Drake's unified method [3]. Our term energies are in very good agreement with the relativistic many-body perturbation theory (MBPT) results of Johnson and Sapirstein [4]. Discrepancy with Drake's results is due largely to the neglect of higher-order relativistic corrections in Drake's calculation. Our results on fine structure splittings and transition energies agree very well with experiment throughout the periodic table.

We have also calculated the term energies for the $n=1,2$ singlet states of heliumlike ions. In this case, QED corrections, including screening and relaxation effects, are calculated with a scheme by Cheng et al. [5] instead of taken from Drake's calculation [3]. For the $K_{\alpha}$ x-ray energies, differences between our results and Drake's results for low-Z to mid-Z ions are due mainly to the relaxation effects in QED corrections which are not included in Drake's calculation. Including relaxation effects in QED corrections improves the agreement between theory and experiment. Residual differences are likely due to higher-order QED corrections not yet considered in any theoretical calculations.

In summary, we found that the relativistic CI method with a complete, finite basis set is an effective way of treating the relativistic correlation energy problem. Extensions of these techniques to treat more complex many-electron ions are now being developed.

1. W. R. Johnson, S. A. Blundell, and J. Sapirstein, Phys. Rev. A 37, 307 (1988).

2. E. R. Davidson, J. Comp. Phys. 17, 87 (1975).

3. G. W. F. Drake, Can. J. Phys. 66, 586 (1988).

4. W. R. Johnson and J. Sapirstein, Phys. Rev. A 46, R2197 (1992).

5. K. T. Cheng, W. R. Johnson, and J. Sapirstein, Phys. Rev. Lett. 66, 2960 (1991); Phys. Rev. A 47, 1817 (1993). 


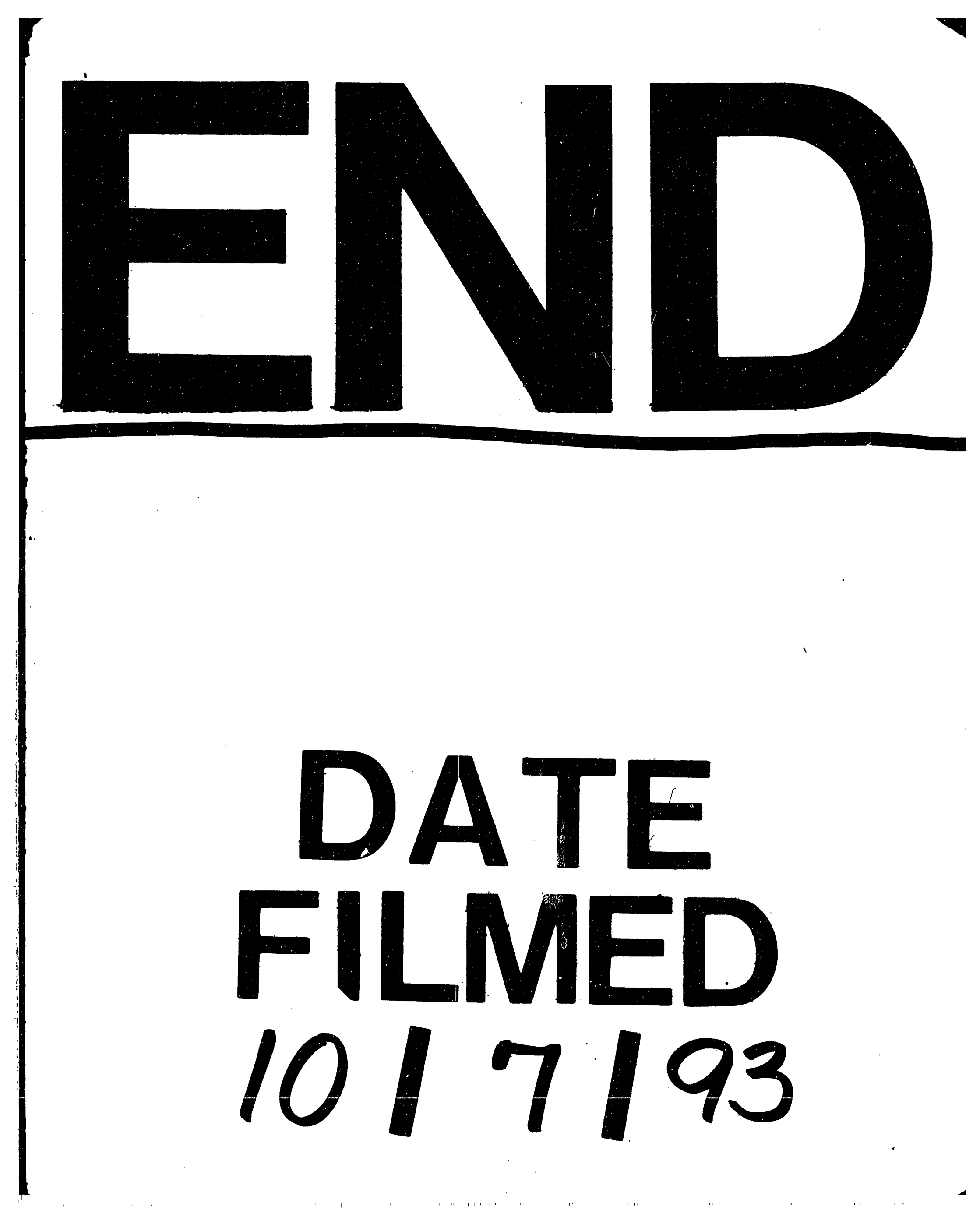



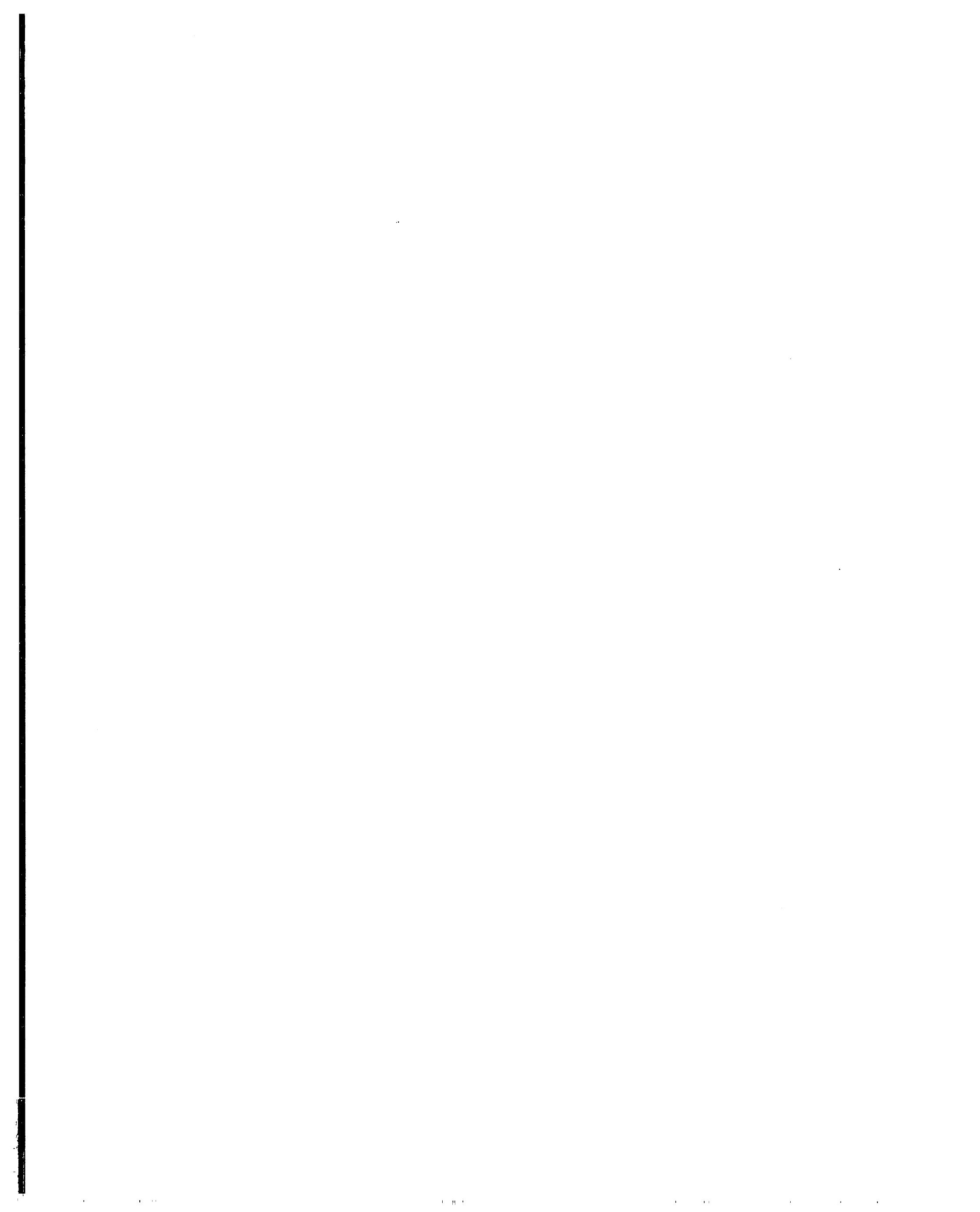